\title{
On Isomorphism Classes of Low Dimensional Non-Lie Filiform Leibniz Algebras over $\mathbb{Z}_{p}$
}

\author{
Nur Syafiqah Edora Ariffin ${ }^{1}$, Sharifah Kartini Said Husain ${ }^{1,2 *}$ and Siti Hasana Sapar ${ }^{1,2}$ \\ ${ }^{1}$ Institute for Mathematical Research, Universiti Putra Malaysia, 43400 UPM Serdang Selangor, Malaysia \\ ${ }^{2}$ Department of Mathematics, Universiti Putra Malaysia, 43400 UPM Serdang Selangor, Malaysia
}

\begin{abstract}
The paper aims to investigate the isomorphism classes of low dimensional filiform Leibniz algebras over some classes of finite field. There are two sources to get the isomorphism classes of filiform Leibniz algebras. The first of them is the naturally graded non-Lie filiform Leibniz algebras and the other one is the naturally graded filiform Lie algebras. This paper deals with the isomorphism classes of filiform Leibniz algebras appearing from the naturally graded non-Lie filiform Leibniz algebras called non-Lie filiform Leibniz algebras. The complete list of isomorphism classes in term of invariant functions of lowdimensional non-Lie filiform Leibniz algebras over $\mathbb{Z}_{p}$ for $p \leq 5$ will be provided.
\end{abstract}

Keywords: Filiform Leibniz algebra, isomorphism classes, invariant function.

\section{INTRODUCTION}

Leibniz algebras were introduced by J-L Today as a "nonantisymmetric" generalization of Lie algebras (Loday, 1993). Leibniz algebras appear to be related in a natural way to several topics such as classical algebraic topology, quantum physics, homological algebra and many more (Albeverio et al., 2006).

Isomorphism criteria of any class of algebras are very theoretical and difficult to solve. The classification of any class of algebras is important in order to study the structure of a member of the class itself. For the case of complex Leibniz algebras, the problems of classification have been solved for dimension up to ten (Albeverio et al., 2006; Ayupov \& Omirov, 1999; Rikhsiboev and Rakhimov, 2012; Khudoyberdiyevet et al., 2014; Rakhimov and Said Husain, 2011; Said Husain, 2011; Sozan et al., 2010; Mohamed et al., 2014; Deraman et al.,
2011; Mohd Kasim et al., 2014; Rakhimov \& Said Husain, 2011; Said Husain et al., 2010; Abdulkareem et al.,2015). Some of the classifications are based on solvability (Khudoyberdiyevet et al., 2014), nilpotentcy (Albeverio et al., 2006) and filiform (Rakhimov and Said Husain, 2011; Said Husain, 2011; Sozan et al., 2010; Mohamed et al., 2014; Deraman et al., 2011; Mohd Kasim et al., 2014; Rakhimov \& Said Husain, 2011; Said Husain et al., 2010; Abdulkareem et al., 2015).

Besides the classification of algebras over complex field, there are also classifications over a special field which is $p$-adic (has zero characteristic). The classifications of filiform Leibniz algebras over $p$-adic have been done for the dimension up to eight (Ayupov and Kurbanbaev, 2010; Ladra et al., 2013; Khudoyberdiyev et al., 2010). Other than that Rakhimov et al., (2018) classified three dimensional Leibniz algebras over arbitrary field $\mathbb{K}$ where $\mathbb{K}=\mathbb{R}, \mathbb{Z}_{3}, \mathbb{Z}_{5}$ and $\mathbb{Z}_{7}$. In this 
paper,classification of four and five dimensional non-Lie filiform Leibniz algebras over $\mathbb{Z}_{p}$ where $p=2,3,5$ will be describe. In section II, basic concept of Leibniz algebras will be given. In section III, we give the isomorphism criteria and complete classification for low dimensional non-Lie filiform Leibniz algebras over $\mathbb{Z}_{p}$.

\section{PRELIMINARIES}

In this section, definitions and theorems will be given and used to deal with the classification problem.

Definition 1.(Said Husain et al., 2010)A Leibniz algebra is a vector space over $\mathbb{F}$, equipped with a $\mathbb{F}$-bilinear map $[\because]:, L \times L \rightarrow L$ satisfying the Leibniz identity $[[x, y], z]=$ $[[x, z], y]+[x,[y, z]]$ for all $x, y, z \in L$.

Let $L$ be a Leibniz algebra. The lower central series is defined as $\mathrm{L}^{1}=\mathrm{L}$ and $\mathrm{L}^{k+1}=\left[\mathrm{L}^{\mathrm{k}}, \mathrm{L}\right]$ where $\mathrm{k} \in \mathbb{N}$. Then, descending series can be written as $\mathrm{L}^{1} \supset \mathrm{L}^{2} \supset \cdots \supset \mathrm{L}^{\mathrm{r}} \supset$ ‥ A Leibniz algebra $L$ is said to be nilpotent if there exists an integer $r \in \mathbb{N}$ such that $\mathrm{L}^{\mathrm{r}}=\{0\}$.

Definition 2.(Mohamed et al., 2014)An n-dimensional Leibniz algebra $L$ is said to be filiform if $\operatorname{dim} L^{i}=n-i$, where $2 \leq i \leq n$.

Filiform Leibniz algebras appeared from two sources which are naturally graded non-Lie filiform Leibniz algebras and naturally graded filiform Lie algebras. In this paper, the class of non-Lie filiform Leibniz algebras which is arising from naturally graded non-Lie filiform Leibniz algebras is considered. From the definition and theorem in (Rakhimov and Said Husain, 2011; Said Husain, 2011; Ayupov and Kurbanbaev 2010) we have the following definition and theorem in the field of $\mathbb{Z}_{p}$.

Theorem 1.In an arbitrary n-dimensional non-Lie filiform Leibniz algebra over $\mathbb{Z}_{p}$, there exists a basis $\left\{e_{1}, e_{2}, \cdots, e_{n}\right\}$ such that the multiplication in this basis has one of thefollowing forms:

$$
\begin{aligned}
& \text { 1. } F L b_{n, p}: \\
& {\left[e_{1}, e_{1}\right]=e_{3},\left[e_{i}, e_{1}\right]=e_{i+1}, \quad 2 \leq i \leq n-1,} \\
& {\left[e_{1}, e_{2}\right]=\alpha_{4} e_{4}+\alpha_{5} e_{5}+\cdots+\alpha_{n-1} e_{n-1}+\theta e_{n},}
\end{aligned}
$$

$$
\begin{aligned}
& {\left[e_{j}, e_{2}\right]=\alpha_{4} e_{j+2}+\alpha_{5} e_{j+3}+\cdots+\alpha_{n+2-j} e_{n}, \quad 2 \leq \mathrm{j}} \\
& \leq \mathrm{n}-2, \\
& \text { 2. } \quad S L b_{n, p}: \\
& \quad\left[e_{1}, e_{1}\right]=e_{3},\left[e_{i}, e_{1}\right]=e_{i+1}, \quad 3 \leq i \leq n-1, \\
& \quad\left[e_{1}, e_{2}\right]=\beta_{4} e_{4}+\beta_{5} e_{5}+\cdots+\beta_{n} e_{n}, \\
& {\left[e_{2}, e_{2}\right]=\gamma e_{n}, \quad 3 \leq \mathrm{j}} \\
& {\left[e_{j}, e_{2}\right]=\beta_{4} e_{j+2}+\beta_{5} e_{j+3}+\cdots+\beta_{n+2-j} e_{n},} \\
& \leq \mathrm{n}-2,
\end{aligned}
$$

where $\alpha_{i}, \theta, \beta_{i}, \gamma \in \mathbb{Z}_{p}$ and the omitted products are being zero.

An adapted transformation can be represented as a product of the elementary basis changing. Definition 3 below will be used in order to get the basis change. Basis transformation $\vartheta$ will be used for $F L b_{n}$ while $\delta$ will be used for $S L b_{n}$.

Definition 3.Define the following types of basis transformation:

$\vartheta(a, b)=\left\{\begin{aligned} f\left(e_{1}\right) & =a e_{1}+b e_{2}, \\ f\left(e_{2}\right) & =(a+b) e_{2}+b\left(\theta-\alpha_{n}\right) e_{n-1}, \\ f\left(e_{i+1}\right) & =\left[f\left(e_{i}\right), f\left(e_{1}\right)\right], 2 \leq i \leq n-1, \\ f\left(e_{3}\right) & =\left[f\left(e_{1}\right), f\left(e_{1}\right)\right], a(a+b) \neq 0,\end{aligned}\right.$

$\delta(a, b, d)=\left\{\begin{aligned} f\left(e_{1}\right) & =a e_{1}+b e_{2}, \\ f\left(e_{2}\right) & =d e_{2}-\frac{b d \gamma}{a} e_{n-1}, \\ f\left(e_{i+1}\right) & =\left[f\left(e_{i}\right), f\left(e_{1}\right)\right], 3 \leq i \leq n-1, \\ f\left(e_{3}\right) & =\left[f\left(e_{1}\right), f\left(e_{1}\right)\right], a d \neq 0,\end{aligned}\right.$

wherea, $b, d \in \mathbb{Z}_{p}$.

\section{RESULTS AND DISCUSSIONS}

This section is divided into two subsections. For the first subsection, the isomorphism criteria for four and five dimensional non-Lie filiform Leibniz algebras over $\mathbb{Z}_{p}$ will be provided while for the second subsection, the complete isomorphism classes for four and five dimensional filiform Leibniz algebras over $\mathbb{Z}_{p}$ where $p=2,3,5$ will be described. 
A. Isomorphism Criteria for non-Lie

filiform Leibniz algebras over $\mathbb{Z}_{p}$.

The following theorems show the isomorphism criterion for $F L b_{n}$ and $S L b_{n}$ for dimensions four and five.

\section{Theorem 2.}

1. Algebras $L_{1}$ and $L_{2}$ from $F L b_{4, p}$ are isomorphic if and only if there exist $a, b \in \mathbb{Z}_{p}$ such that $a(a+b) \neq 0$ and satisfies the following relations:

$$
\alpha_{4}^{\prime}=\frac{(a+b) \alpha_{4}}{a^{2}} \text { and } \theta^{\prime}=\frac{a \theta+b \alpha_{4}}{a^{2}}
$$

2. Algebras $L_{1}$ and $L_{2}$ from $S L b_{4, p}$ are said to be isomorphic if and only if there exist $a, b, d \in \mathbb{Z}_{p}$ such that $a d \neq 0$, and the following conditions hold:

$$
\beta_{4}^{\prime}=\frac{d\left(a \beta_{4}+b \gamma\right)}{a^{3}} a n d \gamma^{\prime}=\frac{d^{2} \gamma}{a^{3}}
$$

Proof of this theorem present in (Ariffin et al., 2018).

\section{Theorem 3.}

1. Two algebras $L_{1}$ and $L_{2}$ from $F L b_{5, p}$ are isomorphic if and only if there exist $a, b \in \mathbb{Z}_{p}$ such that $a(a+b) \neq 0$ and satisfies the following relations:

$\alpha_{4}^{\prime}=\frac{(a+b) \alpha_{4}}{a^{2}}$

$\alpha_{5}^{\prime}=\frac{(a+b) \alpha_{5}-2 a b \alpha_{4}}{a^{3}}$,

$\theta^{\prime}=\frac{a^{2} \theta+a b \alpha_{5}-2(a+b) b \alpha_{4}^{2}}{a^{4}}$.

2. Two algebras $L_{1}$ and $L_{2}$ from $S L b_{5, p}$ are isomorphic if and only if there exist $a, b, d \in \mathbb{Z}_{p}$ such that $a d \neq 0$, and the following conditions hold:

$\beta_{4}^{\prime}=\frac{d \beta_{4}}{a^{2}}$

$\beta_{5}^{\prime}=\frac{d\left(a \beta_{5}+b \gamma-2 b \beta_{4}^{2}\right)}{a^{4}}$,

$$
\gamma^{\prime}=\frac{d^{2} \gamma}{a^{4}}
$$

Proof. For $F L b_{5, p}$, there exists a basis $\left\{\mathrm{e}_{1}, \mathrm{e}_{2}, \mathrm{e}_{3}, \mathrm{e}_{4}, \mathrm{e}_{5}\right\}$ and from Theorem 1 we have the following algebras in $F L b_{5, p}$ :

$$
\begin{gathered}
{\left[e_{1}, e_{1}\right]=e_{3}, \quad\left[e_{1}, e_{2}\right]=\alpha_{4} e_{4}+\theta e_{5}, \quad\left[e_{2}, e_{1}\right]=e_{3},} \\
{\left[e_{2}, e_{2}\right]=\alpha_{4} e_{4}+\alpha_{5} e_{5}, \quad\left[e_{3}, e_{1}\right]=e_{4},} \\
{\left[e_{3}, e_{2}\right]=\alpha_{4} e_{5}, \quad\left[e_{4}, e_{1}\right]=e_{5} .}
\end{gathered}
$$

Then, algebras from $F L b_{5, p}$ will undergo basis transformations $\vartheta$ from Definition 3 and have the following forms:

$e_{1}^{\prime}=a e_{1}+b e_{2}$

$e_{2}^{\prime}=(a+b) e_{2}+b\left(\theta-\alpha_{5}\right) e_{4}$,

$e_{3}^{\prime}=a(a+b) e_{3}+b(a+b) \alpha_{4} e_{4}+b\left(a \theta+b \alpha_{5}\right) \mathrm{e}_{5}$

$e_{4}^{\prime}=a^{2}(a+b) e_{4}+2 a b(a+b) \alpha_{4} e_{5}$,

$e_{5}^{\prime}=a^{3}(a+b) e_{5}$,

where $a^{3}(a+b) \neq 0$ and $a, b, \alpha_{4}, \alpha_{5}, \theta \in \mathbb{Z}_{p}$.

After these algebras undergo basis transformation, we compute them into a new table of multiplication respect to the new basis $\left\{\mathrm{e}_{1}^{\prime}, \mathrm{e}_{2}^{\prime}, \mathrm{e}_{3}^{\prime}, \mathrm{e}_{4}^{\prime}, \mathrm{e}_{5}^{\prime}\right\}$ and obtain the following system of equations:

$$
\begin{aligned}
\alpha_{4}^{\prime}\left(a^{2}(a+b)\right) & =a(a+b) \alpha_{4}+b(a+b) \alpha_{4}, \\
(a+b)^{2} \alpha_{5} & =2 a b(a+b) \alpha_{4}+\alpha_{5}^{\prime} a^{3}(a+b), \\
a \theta+b \alpha_{5} & =2 a b \alpha_{4} \alpha_{4}^{\prime}+\theta^{\prime} a^{3},
\end{aligned}
$$

and can be expressed as follow

$$
\begin{aligned}
& \alpha_{4}^{\prime}=\frac{(a+b) \alpha_{4}}{a^{2}}, \\
& \alpha_{5}^{\prime}=\frac{(a+b) \alpha_{5}-2 a b \alpha_{4}}{a^{3}}, \\
& \theta^{\prime}=\frac{a^{2} \theta+a b \alpha_{5}-2 b(a+b) \alpha_{4}^{2}}{a^{3}} .
\end{aligned}
$$

Thus we proved Theorem 3 for $F L b_{5, p}$.

Note that proof for $S L b_{5, p}$ in Theorem 3 is similar with $F L b_{5, p}$.

We represent the algebras for four dimensional $F L b_{4, p}$ as $L\left(\alpha_{4}, \theta\right)$ and $S L b_{4, p}$ as $L\left(\beta_{4}, \gamma\right)$. Meanwhile the algebras for $F L b_{5, p}$ and $S L b_{5, p}$ of five dimensional will be written as $L\left(\alpha_{4}, \alpha_{5}, \theta\right)$ and $L\left(\beta_{4}, \beta_{5}, \gamma\right)$ respectively. 


\section{B. Isomorphism Classes of Low}

\section{Dimensional Non-Lie Filiform Leibniz}

\section{Algebras over $\mathbb{Z}_{p}$}

In this subsection, the isomorphism classes of non-Lie filiform Leibniz algebras over $\mathbb{Z}_{p}$ in dimensions four and five are provided.

Theorem 4. Let $L$ be an element of $F L b_{4, p}$ or $S L b_{4, p}$. Then $L$ is said to be isomorphic to one of the following pairwise nonisomorphic Leibniz algebras:

\begin{tabular}{|c|c|c|c|}
\hline & $\mathbb{Z}_{p}$ & Representative & \\
\hline \multirow{10}{*}{$F L b_{4, p}$} & \multirow{2}{*}{$p=2$} & $L_{1}(1, \lambda)$ & \multirow{2}{*}{$\lambda \in \mathbb{Z}_{2}$} \\
\hline & & $L_{2}(0, \lambda)$ & \\
\hline & \multirow{3}{*}{$p=3$} & $L_{1}(1, \lambda)$ & \multirow{3}{*}{$\lambda \in \mathbb{Z}_{3}$} \\
\hline & & $L_{2}(2, \lambda)$ & \\
\hline & & $L_{3}(0, \lambda)$ & \\
\hline & \multirow{5}{*}{$p=5$} & $L_{1}(0, \lambda)$ & \multirow{5}{*}{$\lambda \in \mathbb{Z}_{5}$} \\
\hline & & $L_{2}(1, \lambda)$ & \\
\hline & & $L_{3}(2, \lambda)$ & \\
\hline & & $L_{4}(3, \lambda)$ & \\
\hline & & $L_{5}(4, \lambda)$ & \\
\hline \multirow{10}{*}{$S L b_{4, p}$} & \multirow{2}{*}{$p=2$} & $L_{3}(\lambda, 1)$ & \multirow{2}{*}{$\lambda \in \mathbb{Z}_{2}$} \\
\hline & & $L_{4}(\lambda, 0)$ & \\
\hline & \multirow{3}{*}{$p=3$} & $L_{4}(\lambda, 1)$ & \multirow{3}{*}{$\lambda \in \mathbb{Z}_{3}$} \\
\hline & & $L_{5}(\lambda, 2)$ & \\
\hline & & $L_{6}(\lambda, 0)$ & \\
\hline & \multirow{5}{*}{$p=5$} & $L_{6}(\lambda, 0)$ & \multirow{5}{*}{$\lambda \in \mathbb{Z}_{5}$} \\
\hline & & $L_{7}(\lambda, 1)$ & \\
\hline & & $L_{8}(\lambda, 2)$ & \\
\hline & & $L_{9}(\lambda, 3)$ & \\
\hline & & $L_{10}(\lambda, 4)$ & \\
\hline
\end{tabular}

Proving for this Theorem 4 can be referred in (Ariffin et al., 2018).

Theorem 5.The isomorphism classes offive dimensional non-Lie filiform Leibniz algebras over $\mathbb{Z}_{p}$ where $p=$ 2,3,5 can be represented in Table 1 .

Proof. Firstly, for $F L b_{5,2}$. From Theorem 3, since the field is $\mathbb{Z}_{2}$, then from (3) we obtain the following isomorphism criteria.

$\alpha_{4}^{\prime}=\frac{(a+b) \alpha_{4}}{a^{2}}$,

$\alpha_{5}^{\prime}=\frac{(a+b) \alpha_{5}}{a^{3}}$,

$\theta^{\prime}=\frac{a^{2} \theta+a b \alpha_{5}}{a^{4}}$.

Know that $a(a+b) \neq 0$ and $a \neq 0$. Since $\{\mathrm{a}, \mathrm{b}\} \in \mathbb{Z}_{2}$ then we have $a=1$ and $b=0$.

From (5), we obtain

$$
\alpha_{4}^{\prime}=\alpha_{4}, \quad \alpha_{5}^{\prime}=\alpha_{5}, \quad \theta^{\prime}=\theta
$$

Now we consider two cases where $\alpha_{4}=0$ and $\alpha_{4} \neq 0$ and again two cases where $\alpha_{5}=0$ and $\alpha_{5} \neq 0$. As a result, we get $L_{1}(0,0, \lambda), L_{2}(0,1, \lambda), L_{3}(1,0, \lambda)$ and $L_{4}(1,1, \lambda)$ where $\lambda=\theta \in \mathbb{Z}_{2}$.

Next, for $F L b_{5,3}$ where the field is $\mathbb{Z}_{3}$. The system of equation (3) becomes:

$\alpha_{4}^{\prime}=\frac{(a+b) \alpha_{4}}{a^{2}}$,

$\alpha_{5}^{\prime}=\frac{(a+b) \alpha_{5}+a b \alpha_{4}}{a^{3}}$,

$\theta^{\prime}=\frac{a^{2} \theta+a b \alpha_{5}+b(a+b) \alpha_{4}^{2}}{a^{4}}$.

Here, four cases are to be considered where $(a, b)=$ $\{(1,0),(1,1),(2,0),(2,2)\}$.

For case $(a, b)=(1,0)$. From (6), we have the following relation:

$$
\alpha_{4}^{\prime}=\alpha_{4}, \alpha_{5}^{\prime}=\alpha_{5}, \quad \theta^{\prime}=\theta .
$$

As a result, nine algebras are obtained which are $L_{1}(0,0, \lambda), L_{2}(0,1, \lambda), L_{3}(0,2, \lambda), L_{4}(1,0, \lambda), L_{5}(1,1, \lambda)$, $L_{6}(1,2, \lambda), L_{7}(2,0, \lambda), L_{8}(2,1, \lambda)$ and $L_{9}(2,2, \lambda)$ where the value of $\lambda \in \mathbb{Z}_{3}$.

For thesecond case where $(\mathrm{a}, \mathrm{b})=(1,1)$, system (6) becomes:

$$
\alpha_{4}^{\prime}=2 \alpha_{4}, \alpha_{5}^{\prime}=2 \alpha_{5}+\alpha_{4}, \theta^{\prime}=\theta+\alpha_{5}+2 \alpha_{4}^{2} .
$$

Then, we consider case by case and get the same algebras as in the case $(a, b)=(1,0)$.

Next, the third case is $(a, b)=(2,0)$. We get the following isomorphism criteria 


$$
\alpha_{4}^{\prime}=2 \alpha_{4}, \quad \alpha_{5}^{\prime}=\alpha_{5}, \quad \theta^{\prime}=\theta
$$

The algebras obtained from this case are equivalent to the case $(a, b)=\{(1,0),(1,1)\}$.

Lastly for the case $(a, b)=(2,1)$, we have:

$$
\alpha_{4}^{\prime}=\alpha_{4}, \quad \alpha_{5}^{\prime}=\frac{\alpha_{5}+\alpha_{4}}{2}, \quad \theta^{\prime}=\theta+\alpha_{5}+2 \alpha_{4}^{2} .
$$

In this case we only obtain six algebras since the values of $\alpha_{5}$ is limited as we need $\alpha_{5}^{\prime} \in \mathbb{Z}_{3}$. The algebras $L_{1}(0,0, \lambda)$ where $\lambda=\theta \in \mathbb{Z}_{3}, L_{2}(0,1, \lambda)$ where $\lambda=\theta+2 \in$ $\mathbb{Z}_{3}, L_{4}(1,0, \lambda)$ where $\lambda=\theta+1 \in \mathbb{Z}_{3}, L_{5}(1,1, \lambda)$ where $\lambda=$ $\theta \in \mathbb{Z}_{3}, L_{7}(2,0, \lambda)$ where $\lambda=\theta \in \mathbb{Z}_{3}$ and $L_{8}(2,1, \lambda)$ where $\lambda=\theta+2 \in \mathbb{Z}_{3}$ are obtained.

Lastly, for $F L b_{5,5}$, we get the isomorphism criteria as follows:

$\alpha_{4}^{\prime}=\frac{(a+b) \alpha_{4}}{a^{2}}$,

$\alpha_{5}^{\prime}=\frac{(a+b) \alpha_{5}+3 a b \alpha_{4}}{a^{3}}$,

$\theta^{\prime}=\frac{a^{2} \theta+a b \alpha_{5}+3 b(a+b) \alpha_{4}^{2}}{a^{4}}$.

In $F L b_{5,5}$, we consider four cases where $(\mathrm{a}, \mathrm{b})=$ $\{(1,\{0,1,2,3\}),(2,\{0,1,2,4\}),(3,\{0,1,3,4\}),(4,\{0,2,3,4\})\}$. As usual, in order to obtain the algebras, we solve them case by case. Throughout the calculation, we observe that in $\mathbb{Z}_{5}$, we have a total of 25 algebras (see in Table 1 ).

Secondly, proving for $S L b_{5, p}$ where $p=2,3,5$ is quite similar with $F L b_{5, p}$. The only differences are the condition in Theorem 5 where $a d \neq 0$ and the isomorphism criteria for each $\mathbb{Z}_{p}$.

In $S L b_{5,2}$, we get the isomorphism criteria as follow:

$$
\beta_{4}^{\prime}=\frac{d \beta_{4}}{a^{2}}, \beta_{5}^{\prime}=\frac{d\left(a \beta_{5}+b \gamma\right)}{a^{4}}, \gamma^{\prime}=\frac{d^{2} \gamma}{a^{4}} .
$$

Meanwhile in $\mathbb{Z}_{3}$, the following relations are obtain:

$$
\beta_{4}^{\prime}=\frac{d \beta_{4}}{a^{2}}, \beta_{5}^{\prime}=\frac{d\left(a \beta_{5}+b \gamma+\beta_{4}^{2}\right)}{a^{4}}, \gamma^{\prime}=\frac{d^{2} \gamma}{a^{4}} .
$$

Lastly, in $\mathbb{Z}_{5}$, we have the following systems:

$$
\begin{gathered}
\beta_{4}^{\prime}=\frac{d \beta_{4}}{a^{2}}, \\
\beta_{5}^{\prime}=\frac{d\left(a \beta_{5}+3 b \gamma+3 \beta_{4}^{2}\right)}{a^{4}}, \\
\gamma^{\prime}=\frac{d^{2} \gamma}{a^{4}} .
\end{gathered}
$$

\section{CONCLUSION}

As dimension increase, the number of algebras for each $\mathbb{Z}_{p}$ also increase.

Conjecture: The number of isomorphism classes of $n$-dimensional Leibniz algebra over $\mathbb{Z}_{p}$ is $2 p^{n-3}$ where $n \geq$ 4.

\section{ACKNOWLEDGMENT}

This research was supported by Grant 01-01-18-2032FR and sponsored by UPM's Graduate Research Fellowship (GRF) scheme. 


\section{REFERENCES}

Abdulkareem, A. O., Rakhimov, I. S., and Said Husain, S. K. (2015). Isomorphism classes and invariants of low dimensional filiform Leibniz algebras, Journal of Linear and Multilinear algebra, 63(11), 2254-2274.

Albeverio, S., Omirov, B. A., and Rakhimov, I. S. (2006). Classification of 4-dimensional nilpotent complex leibniz algebras. Extracta Math., 21(3), 197-210.

Ariffin, N. S. E., Said Husain, S. K., Sapar, S. H. and Nawawi, A. (2018). The classification of 4-dimensional non-Lie filiform Leibniz algebras over finite field, $\mathbb{Z}_{p}$. Jurnal Karya Asli Lorekan Ahli Matematik, 11(1), o38049.

Ayupov, S. A., and Kurbanbaev, T. K. (2010). The classification of 4 -dimensional $p$-adic filiform Leibniz algebras. TWMS Journal of Pure and Applied Mathematics, 1(2), 155-162.

Ayupov, S. A., and Omirov B. A. (1999). On 3 dimensional Leibniz algebras, Uzbek Math. Jour., 1, 9-14.

Deraman, F., Rakhimov, I. S., and Said Husain, S. K. (2011). Isomorphism classes and invariants for a subclass of nine-dimensional filiform Leibniz algebras,

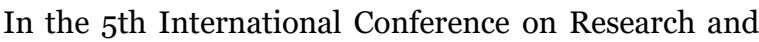
Education in Mathematics (eds E.T. Baskoro and D. Suprijanto), 326-331.

Khudoyberdiyev, A. Kh., Kurbanbaev, T. K. and Omirov, B. A. (2010). Classification of three-dimensional solvable $p$-adic Leibniz algebras. $p$-Adic Numbers Ultrametric Anal. Appl. 2(3), 207-221.

Khudoyberdiyev, A. Kh., Rakhimov, I. S., and Said Husain, S. K. (2014). On classification of 5 dimensional solvable Leibniz algebras, Journal of Linear algebra and its Applications, 457, 428-454.

Ladra, M., Omirov, B. A. and Rozikov, U. A. (2013). Classification of $p$-Adic 6-Dimensional Filiform Leibniz Algebras by Solution of $x^{q}=a$. Central European Journal of Mathematics. 11(6), 1083-1093.

Loday, L. J. (1993). Une version non commutative des algebres de Lie: les algebres de Leibniz, Enseign. Math., 39(3-4), 269-293.

Mohamed, N. S., Said Husain, S. K., and Rakhimov, I. S.
(2012). On classification for first class of 10 dimensional of complex filiform Leibniz algebras. In Proceedings of International Sciences Postgraduate Conference (ISPC2012), 487-498. (Johor Bharu, Malaysia.)

Mohamed, N. S., Said Husain, S. K., and Rakhimov, I. S. (2014). On isomorphism classes of a subclass of filiform Leibniz algebras in dimensional 10. AIP Conf. Proc., 1602, 736-743.

Mohd Kasim, S., Rakhimov, I. S., and Said Husain, S. K. (2014). Isomorphism classes of 10-dimensional filiform Leibniz algebras. AIP Conf. Proc., 1602, 708715 .

Rakhimov, I. S., and Said Husain, S. K. (2011). Classification of a subclass of nilpotent Leibniz algebras, Journal of Linear and Multilinear algebra, 59(3), 339-354.

Rakhimov, I. S., and Said Husain, S. K. (2011). On isomorphism classes and invariants of low dimensional complex filiform Leibniz algebras, Journal of Linear and Multilinear algebra, 59(2), 205220.

Rakhimov, I. S., Rikhsiboev, I. M. And Mohammed, M. A. (2018). An algorithm for classification of threedimensional Leibniz algebras over arbitrary fields. JP Journal of Algebra, Number Theory and Applications. 4, 181-198.

Rikhsiboev, I. M., and Rakhimov, I. S. (2012). Classification of three dimensional complex Leibniz algebras, AIP Conf. Proc., 1450, 358, d

Said Husain, S. K. (2011). Classification of Non Lie Complex Filiform Leibniz Algebras for Low Dimensions. Doctor of Philosophy Thesis, 23.

Said Husain, S. K., Hassan, M. A., and Rakhimov, I. S. (2010). On isomorphisms and invariants of $5^{\text {th }}$ dimensional complex filiform Leibniz algebras, AIP Conference Proceedings, 1309, 430-438.

Sozan, J. O., Rakhimov, I. S., and Kamal Atan, A. M. (2010). Classification of first class of complex filiform Leibniz algebras. Lampert Academi Publishing. 
TABLE 1: Isomorphism classes of five dimensional non-Liefiliform Leibniz algebras over $\mathbb{Z}_{P}$.

\begin{tabular}{|c|c|c|c|c|c|}
\hline \multirow{2}{*}{$\mathbb{Z}_{p}$} & \multicolumn{4}{|c|}{ Representative } & \\
\hline & \multicolumn{2}{|c|}{$F L b_{5, p}$} & \multicolumn{2}{|c|}{$S L b_{5, p}$} & \\
\hline \multirow{2}{*}{$p=2$} & $L_{1}(0,0, \lambda)$ & $L_{2}(0,1, \lambda)$ & $L_{5}(0, \lambda, 0)$ & $L_{6}(0, \lambda, 1)$ & \multirow{2}{*}{$\lambda \in \mathbb{Z}_{2}$} \\
\hline & $L_{3}(1,0, \lambda)$ & $L_{4}(1,1, \lambda)$ & $L_{7}(1, \lambda, 0)$ & $L_{8}(1, \lambda, 1)$ & \\
\hline \multirow{5}{*}{$p=3$} & $L_{1}(0,0, \lambda)$ & $L_{2}(0,1, \lambda)$ & $L_{10}(0, \lambda, 0)$ & $L_{11}(0, \lambda, 1)$ & \multirow{5}{*}{$\lambda \in \mathbb{Z}_{3}$} \\
\hline & $L_{3}(0,2, \lambda)$ & $L_{4}(1,0, \lambda)$ & $L_{12}(0, \lambda, 2)$ & $L_{13}(1, \lambda, 0)$ & \\
\hline & $L_{5}(1,1, \lambda)$ & $L_{6}(1,2, \lambda)$ & $L_{14}(1, \lambda, 1)$ & $L_{15}(1, \lambda, 2)$ & \\
\hline & $L_{7}(2,0, \lambda)$ & $L_{8}(2,1, \lambda)$ & $L_{16}(2, \lambda, 0)$ & $L_{17}(2, \lambda, 1)$ & \\
\hline & \multicolumn{2}{|c|}{$L_{9}(2,2, \lambda)$} & \multicolumn{2}{|c|}{$L_{18}(2, \lambda, 2)$} & \\
\hline \multirow{13}{*}{$p=5$} & $L_{1}(0,0, \lambda)$ & $L_{2}(0,1, \lambda)$ & $L_{26}(0, \lambda, 0)$ & $L_{27}(0, \lambda, 1)$ & \multirow{13}{*}{$\lambda \in \mathbb{Z}_{5}$} \\
\hline & $L_{3}(0,2, \lambda)$ & $L_{4}(0,3, \lambda)$ & $L_{28}(0, \lambda, 2)$ & $L_{29}(0, \lambda, 3)$ & \\
\hline & $L_{5}(0,4, \lambda)$ & $L_{6}(1,0, \lambda)$ & $L_{30}(0, \lambda, 4)$ & $L_{31}(1, \lambda, 0)$ & \\
\hline & $L_{7}(1,1, \lambda)$ & $L_{8}(1,3, \lambda)$ & $L_{32}(2, \lambda, 0)$ & $L_{33}(3, \lambda, 0)$ & \\
\hline & $L_{9}(1,4, \lambda)$ & $L_{10}(2, O, \lambda)$ & $L_{34}(4, \lambda, O)$ & $L_{35}(1, \lambda, 1)$ & \\
\hline & $L_{11}(2,1, \lambda)$ & $L_{12}(2,3, \lambda)$ & $L_{36}(1, \lambda, 2)$ & $L_{37}(1, \lambda, 3)$ & \\
\hline & $L_{13}(2,4, \lambda)$ & $L_{14}(3,0, \lambda)$ & $L_{38}(1, \lambda, 4)$ & $L_{39}(2, \lambda, 1)$ & \\
\hline & $L_{15}(3,1, \lambda)$ & $L_{16}(3,3, \lambda)$ & $L_{40}(2, \lambda, 2)$ & $L_{41}(2, \lambda, 3)$ & \\
\hline & $L_{17}(3,4, \lambda)$ & $L_{18}(4,0, \lambda)$ & $L_{42}(2, \lambda, 4)$ & $L_{43}(3, \lambda, 1)$ & \\
\hline & $L_{19}(4,1, \lambda)$ & $L_{20}(4,3, \lambda)$ & $L_{44}(3, \lambda, 2)$ & $L_{45}(3, \lambda, 3)$ & \\
\hline & $L_{21}(4,4, \lambda)$ & $L_{22}(1,2, \lambda)$ & $L_{46}(3, \lambda, 4)$ & $L_{47}(4, \lambda, 1)$ & \\
\hline & $L_{23}(2,2, \lambda)$ & $L_{24}(3,2, \lambda)$ & $L_{48}(4, \lambda, 2)$ & $L_{49}(4, \lambda, 3)$ & \\
\hline & \multicolumn{2}{|c|}{$L_{25}(4,2, \lambda)$} & \multicolumn{2}{|c|}{$L_{50}(4, \lambda, 4)$} & \\
\hline
\end{tabular}

